ORIGINAL ARTICLE

\title{
Prospective examination of adolescent sleep patterns and behaviors before and during COVID-19
}

\author{
Stephen P. Becker ${ }^{1,2, *,}$, Melissa R. Dvorsky',4,®, Rosanna Breaux ${ }^{5}$, Caroline N. Cusick $^{6}$, \\ Katherine P. Taylor ${ }^{1}$ and Joshua M. Langberg ${ }^{6}$
}

${ }^{1}$ Division of Behavioral Medicine and Clinical Psychology, Cincinnati Children's Hospital Medical Center, Cincinnati, OH, ${ }^{2}$ Department of Pediatrics, University of Cincinnati College of Medicine, Cincinnati, OH, ${ }^{3}$ Division of Psychology and Behavioral Health, Children's National Hospital, Washington, DC, ${ }^{4}$ Department of Pediatrics, the George Washington University School of Medicine and Health Sciences, Washington, DC, ${ }^{5}$ Department of Psychology, Virginia Polytechnic Institute and State University, Blacksburg, VA and ${ }^{6}$ Department of Psychology, Virginia Commonwealth University, Richmond, VA

*Corresponding author. Stephen Becker, Division of Behavioral Medicine and Clinical Psychology, Cincinnati Children's Hospital Medical Center, 3333 Burnet Avenue, Cincinnati, OH 45229-3039. Email: stephen.becker@cchmc.org.

\begin{abstract}
Study Objectives: To prospectively examine changes in adolescent sleep before and during the COVID-19 pandemic in adolescents with and without ADHD.

Methods: Participants were 122 adolescents (ages 15-17; 61\% male; 48\% with ADHD). Parents reported on adolescents' sleep duration and difficulties initiating and maintaining sleep (DIMS); adolescents reported on sleep patterns, sleep duration, delayed sleep/wake behaviors, and daytime sleepiness before (September 2019 to February 2020) and during (May-June 2020) COVID-19. Adolescents also reported on their health behaviors, COVID-19-related negative affect, and difficulties concentrating due to COVID-19.

Results: Parents reported adolescents had more DIMS during COVID-19 than before COVID-19, with clinically elevated rates increasing from $24 \%$ to $36 \%$. Both bedtimes and waketimes shifted later during COVID-19, and adolescents reported more delayed sleep/wake behaviors. Adolescents also reported less daytime sleepiness and longer school night sleep duration during COVID-19. In considering differences between adolescents with and without ADHD, adolescents with ADHD did not experience an increase in school night sleep duration and were less likely to obtain recommended sleep duration during COVID-19. In the full sample, controlling for ADHD status, COVID-19-related sadness/loneliness was associated with increases in DIMS, and spending less time outside and more COVID-19-related worries/fears were associated with increases in delayed sleep/wake behaviors during COVID-19.

Conclusions: COVID-19 had negative and positive impacts on adolescent sleep. Adolescents with ADHD did not experience the benefit of increased school night sleep duration during COVID-19 like adolescents without ADHD. Negative affect and health behaviors may be useful intervention targets for reducing negative impacts of COVID-19 for adolescent sleep.

\section{Statement of Significance}

The current study documents prospective changes in sleep patterns and behaviors in adolescents before and during the COVID-19 pandemic. In a sample of 122 adolescents, bedtimes and wake times were later, daytime sleepiness was lower, and school night sleep duration was longer during COVID-19 compared to before COVID-19. However, adolescents reported more extreme delayed sleep/wake behaviors during COVID-19, and parents reported that adolescents had more sleep difficulties during COVID-19, with rates of clinically elevated difficulties increasing from 24\% before COVID-19 to 36\% during COVID-19. Given the known benefits of sufficient and high-quality sleep for adolescent health and development, clinicians should assess and monitor sleep when treating adolescents during and after the pandemic.
\end{abstract}

Key words: coronavirus; adolescence; attention-deficit/hyperactivity disorder; health behaviors; negative affect

Submitted: 12 October, 2020; Revised: 19 January, 2021

๑ Sleep Research Society 2021. Published by Oxford University Press on behalf of the Sleep Research Society.

All rights reserved. For permissions, please email: journals.permissions@oup.com 


\section{Introduction}

The COVID-19 pandemic has profoundly impacted daily life, including prolonged social distancing, changes in routines, and increases in stress and psychopathology [1]. Each of these factors alone are associated with poorer sleep under typical circumstances [2], and when combined during a chronic stressor such as the COVID-19 pandemic, are expected to contribute to marked changes in sleep patterns and functioning [3, 4]. These changes may include a shift in bedtimes and wake times, in addition to the possibility of both worsened sleep (e.g. more sleep difficulties) and improved sleep (e.g. longer sleep duration).

Most studies examining sleep in the context of COVID-19 have used adult samples. One prospective [5] and numerous retrospective [6-8] studies have found bedtimes and wake times to be later during COVID-19 compared to before COVID-19. In addition, cross-sectional studies have found $30 \%-70 \%$ of adults to report poor or insufficient sleep during the COVID-19 pandemic [9-13]. However, there have also been some indications of improvements in sleep, with studies finding adults to have longer sleep duration during COVID-19 [5, 8] and for a higher percentage to obtain the recommended amounts of nighttime sleep [5]. Similarly, a qualitative study with 45 adolescents found adolescents to report a delayed sleep schedule (by about 2 hours), improved sleep quality, longer sleep duration, and less daytime sleepiness during COVID-19 compared to before COVID-19 [14].

Many biological and environmental factors make adolescence a unique developmental stage for insufficient and poorquality sleep [15]. Adolescence, particularly during the high school years, is associated with significant changes in sleep/ wake schedules, decreased sleep duration (particularly on school days), later endogenous circadian phase, and increased daytime sleepiness [15-17]. These adolescent sleep patterns contribute to social jetlag, with adolescents often having different sleep-wake schedules on schooldays compared to weekends due to a misalignment of weekday obligations (e.g. school start time) with their circadian clock [15]. The COVID-19 pandemic and its associated remote learning, limited social and extracurricular activities, and social distancing restrictions may especially impact adolescent sleep [4]. In line with this possibility, a cross-sectional study found high school students (and college students) to have poorer sleep than middle school students during COVID-19 [18]. Studies with adults have also found younger adults to have more sleep problems than older adults during COVID-19 [19-21], suggesting that adolescence and early adulthood may be developmental periods particularly susceptible to COVID-19-related changes in sleep.

A key limitation of existing studies is their reliance on retrospective or cross-sectional designs. One of the only prospective longitudinal studies in adults examining sleep before and during COVID-19 demonstrates retrospective reporting is subject to "rosy retrospection" whereby the past is remembered more positively than it actually was [22]. It is thus possible, even likely, that asking individuals to report during COVID-19 on their perceptions of sleep before the COVID-19 pandemic will yield biased results. Yet studies with prospective data are rare, and we are unaware of any studies that have examined sleep in youth before and during the COVID-19 pandemic using a prospective longitudinal design.

Adolescents with pre-existing neurodevelopmental disorders, such as attention-deficit/hyperactivity disorder (ADHD), may be at particular risk for negative impacts during COVID-19 [23], including sleep problems. Adolescents with ADHD experience worse sleep functioning than their peers without ADHD $[24,25]$, are more likely to use technology [26], have difficulties maintaining structure/routines [27], and frequently experience co-occurring mental health symptoms such as depression and anxiety $[28,29]$. Thus far, only two studies have examined sleep functioning during COVID-19 in youth with ADHD. In a sample of 241 youth (ages 6-15) with ADHD in China, $48 \%$ of parents indicated that COVID-19 had not changed their child's sleep, with $20 \%$ indicating that sleep had worsened, and $32 \%$ indicating that sleep had improved [30]. Similarly, in a sample of 213 youth (ages 5-17 years) with ADHD in Australia, on average, parents did not report differences in their child's sleep duration from before to during COVID-19 [31]. However, it should be noted that both studies used retrospective parent-report of a single sleep item, used a broad age range spanning childhood and adolescence, and did not include a comparison sample of youth without ADHD. The present study builds upon these studies by examining both parent- and adolescent-reported sleep, including multiple sleep domains, using a prospective longitudinal design with data collected both before and during COVID-19 in adolescents with and without ADHD.

Finally, if adolescent sleep changes during COVID-19, it is important to understand what factors are associated with these changes. In the current study, we examine physical health and affective/cognitive factors that may be associated with changes in sleep during the COVID-19 pandemic. In terms of physical health-related behaviors, youth have been found to have less physical activity, less outside time, and more screen time during COVID-19 compared to before COVID-19 [30, 32], and it has been hypothesized that these behaviors may contribute to shorter sleep duration or shifts in sleep schedules during the pandemic [4]. The pandemic and associated social distancing guidelines may also contribute to negative affect $[33,34]$ and COVID-19related worries or concentration difficulties [35-37], which can negatively impact sleep [38].

Given this backdrop, the current study used a prospective longitudinal sample of adolescents to examine changes in sleep functioning prior to and during COVID-19 in adolescents with and without ADHD, in addition to exploring correlates of changes in sleep duration and behaviors. There were three objectives:

1. To examine change in categorical measures of the percentage of adolescents not obtaining recommended school night sleep duration (i.e. 8-10 hours per night for adolescents ages 14-17 per the National Sleep Foundation guidelines) [39] and clinically elevated difficulties initiating and maintaining sleep from before to during the pandemic. Consistent with findings of studies in adults [5, 8], it was hypothesized that more adolescents would obtain sufficient sleep duration during the pandemic compared to before the pandemic. Based on research suggesting increased sleep duration during COVID-19 in non-ADHD samples [5, 8], but not in youth with ADHD [31], it was also hypothesized that adolescents with ADHD would be less likely than adolescents without ADHD to obtain recommended sleep duration during the pandemic. Also based on extant studies with adults [9-13], we expected higher rates of clinically elevated difficulties initiating and maintaining sleep during 
the pandemic compared to before the pandemic, particularly for adolescents without ADHD relative to adolescents with ADHD [30].

2. To examine change in dimensional measures of adolescent- and parent-reported sleep patterns and behaviors from within 6 months of the COVID-19 pandemic to during the pandemic, and whether adolescents with and without ADHD differed in changes in sleep. It was hypothesized that there would be an effect of time whereby bedtimes and rise times would be later, sleep duration would be longer on school nights specifically, and sleep behaviors (i.e. difficulties initiating and maintaining sleep, delayed sleep/wake behaviors) would be poorer during the pandemic compared to prior to the pandemic. Given extensive research highlighting sleep problems and significantly shorter sleep duration among adolescents with ADHD relative to their peers [24, 25], it was also hypothesized that there would be an effect of group, with adolescents with ADHD having shorter sleep duration and more delayed sleep/wake behaviors than adolescents without ADHD both before and during the pandemic. Based on extant research [30, 31], it was also hypothesized that adolescents with ADHD would not experience changes in sleep duration or difficulties initiating and maintaining sleep during the pandemic compared to before the pandemic.

3. To evaluate correlates of changes in adolescent sleep duration and delayed sleep/wake behaviors during the pandemic. Specifically, we explored demographic characteristics, health behaviors during COVID-19 (i.e. exercise, spending time outdoors, social media use), COVID-19-related negative affect (i.e. worried/afraid, sad/lonely, frustrated/angry), and difficulties concentrating due to COVID-19 as correlates of changes in sleep duration and behaviors during the pandemic. For these analyses, ADHD group status was included in the models to determine (1) whether ADHD remained associated with changes in adolescent sleep duration and delayed sleep/wake behaviors above and beyond demographic characteristics, health behaviors during COVID-19, COVID-19-related negative affect, and difficulties concentrating due to COVID-19, and (2) whether the correlates of interest were associated with changes in adolescent sleep above and beyond ADHD status.

\section{Methods}

\section{Participants and procedures}

Participants were 122 adolescents (61.5\% male; ages 15-17 years; Mage $=15.73$ years at the pre-COVID-19 assessment and 16.28 years at the COVID-19 assessment) currently in the tenth grade (one participant was in ninth grade due to being retained a grade, and one participant was in eleventh grade due to being accelerated a grade). Participants with and without ADHD were initially recruited in eighth grade from local schools across Ohio, Kentucky, and Virginia (United States). Based on a comprehensive evaluation, 58 of the adolescents were diagnosed with DSM-5 ADHD, with remaining participants $(n=64)$ comprising a comparison sample without ADHD. Adolescents identified as predominantly White (81\%), with 13\% identifying as biracial/ multiracial, $3 \%$ identifying as Asian, and 3\% identifying as Black. Participants came from a range of socioeconomic backgrounds
( $M$ family income $=\$ 93,073, S D=\$ 34,856)$. Of the adolescents with ADHD, 83\% were on medication for ADHD pre-COVID-19. In the total sample, $16 \%$ were on medication for emotional and behavioral disorders (e.g. depression; 23\% in the ADHD sample; $9 \%$ in the comparison sample) pre-COVID-19. Additional details regarding the sample and differences between adolescents with and without ADHD are provided in Table 1.

The larger study included two cohorts [24], though only the second cohort completed a visit within the 6 months prior to the onset of COVID-19 in the United States (September 2019-February 2020). As such, this timepoint is ideally suited to prospectively evaluate the impact of COVID-19 on adolescent sleep functioning during the pandemic, and only participants from the second cohort were included in the current study. Participants from the larger study who provided permission for further contact ( $90.8 \%$ retention) were invited to participate in the current study, with data for the present study collected between May 16 and June 15, 2020. These data were collected when schools had shifted to remote learning and participants were under stay-at-home orders and/or strong recommendations to stay home. Specifically, stay-at-home orders for Ohio were March 23-May 29, stay-at-home orders for Kentucky were March 26-May 22 with additional restrictions through June 29, and stay-at-home orders in Virginia were March 30-June 10. After formal stay-at-home orders were lifted in Ohio, "strong recommendations" for residents to "stay at their place of residence when possible" were issued. After the initial stay-at-home orders, no adolescents returned to in-person schooling for the remainder of the academic year.

Individuals who participated in the COVID-19 timepoint did not differ from individuals who were contacted for possible participation on adolescent sex, race, ethnicity, ADHD symptoms, or family income ( $p s>.05$ ). Additionally, pre-COVID-19 sleep variables did not significantly differ for individuals who did or did not participate in the COVID-19 timepoint ( $p s>.05$ ), with only one exception. Adolescents who did not participate in the COVID-19 timepoint had higher scores $(t=2.12, p=.039)$ on the delayed sleep/wake behaviors measure (described below; $M=8.13, S D=3.52$ ) relative to adolescents who did participate $(\mathrm{M}=6.89, \mathrm{SD}=2.32)$

Adolescents and parents provided consent and assent, respectively, for pre-COVID-19 data collection and again for participation in the COVID-19 data collection. Data at both timepoints was collected online via REDCap, with the preCOVID-19 measures completed either at home or an in-person visit and all measures during COVID-19 completed remotely at home. Adolescents and parents were compensated for participation at both timepoints.

\section{Measures}

\section{Sleep Habits Survey}

The Sleep Habits Survey (SHS) [40] is an adolescent-report measure assessing various domains of sleep functioning that has been validated for youth ages 10-19 years. The SHS was completed at both study timepoints. The SHS includes a 10-item Sleep/Wake Problems subscale, with items are rated on a fivepoint scale ( 1 = never; 5 = every day/night). However, several items relate to tardiness/sleepiness during school (e.g. arrived late to class because you overslept, fallen asleep in a morning class). Because it is unlikely that these items were relevant during 
Table 1. Sample characteristics

\begin{tabular}{|c|c|c|c|c|c|}
\hline & \multirow{2}{*}{$\frac{\text { Total sample }(\mathrm{N}=122)}{\mathrm{M} \pm \mathrm{SD}}$} & \multirow{2}{*}{$\frac{\text { ADHD group }(n=58)}{M \pm S D}$} & \multirow{2}{*}{$\frac{\text { Comparison group }(n=64)}{M \pm S D}$} & \multirow[t]{2}{*}{ Group differences } & \multirow[t]{2}{*}{$P$} \\
\hline & & & & & \\
\hline Age & $16.28 \pm 0.35$ & $16.27 \pm 0.36$ & $16.29 \pm 0.34$ & $t=0.32$ & .750 \\
\hline Family income & $\begin{array}{l}\$ 97,066 \pm 32,810 \\
N(\%)\end{array}$ & $\begin{array}{l}\$ 86,403 \pm 35,478 \\
N(\%)\end{array}$ & $\begin{array}{l}\$ 106,562 \pm 27,151 \\
N(\%)\end{array}$ & $t=3.53$ & .001 \\
\hline Sex & & & & $\chi^{2}=2.62$ & .106 \\
\hline Female & $47(38.5)$ & $18(31.0)$ & $29(45.3)$ & & \\
\hline Male & $75(61.5)$ & $40(69.0)$ & $35(54.7)$ & & \\
\hline Race & & & & $\chi^{2}=1.85$ & .763 \\
\hline White & $103(84.4)$ & $49(84.5)$ & $54(84.4)$ & & \\
\hline Asian & $5(4.1)$ & $2(3.4)$ & $3(4.7)$ & & \\
\hline Black & $5(4.1)$ & $3(5.2)$ & $2(3.1)$ & & \\
\hline American Indian & $1(0.8)$ & $1(1.7)$ & $0(0.0)$ & & \\
\hline Bi/Multiracial & $8(6.6)$ & $3(5.2)$ & $5(7.8)$ & & \\
\hline Ethnicity & & & & $\chi^{2}=0.65$ & .798 \\
\hline Hispanic/Latinx & $7(5.7)$ & $3(5.2)$ & $4(6.3)$ & & \\
\hline Not Hispanic/Latinx & $115(94.3)$ & $55(94.8)$ & $60(93.8)$ & & \\
\hline Other psychiatric diagnoses & $44(36.1)$ & $29(50.0)$ & $15(23.4)$ & $\chi^{2}=9.31$ & .002 \\
\hline Any externalizing (ODD/CD) & $15(12.3)$ & $13(22.4)$ & $2(3.1)$ & $\chi^{2}=10.50$ & .001 \\
\hline Any anxiety & $33(27.0)$ & $19(32.8)$ & $14(21.9)$ & $\chi^{2}=1.83$ & .177 \\
\hline Any depression & $11(9.0)$ & $8(13.8)$ & $3(4.7)$ & $\chi^{2}=3.08$ & .079 \\
\hline
\end{tabular}

Age is at the timepoint during COVID-19. Income information was missing for one family. Comorbidity was assessed at the initial evaluation for the larger study using structured diagnostic interviews with the parent and adolescent (see Becker et al.[24]). ADHD = attention-deficit/hyperactivity disorder. ODD/CD = oppositional defiant disorder/conduct disorder based on a structured diagnostic interview with the adolescent's parent. Any anxiety = presence of generalized anxiety disorder, social phobia, obsessive-compulsive disorder, and/or posttraumatic stress disorder (PTSD) based on a structured diagnostic interview conducted separately with the adolescent and their parent. Any depression = presence of major depression or dysthymia based on a structured diagnostic interview conducted separately with the adolescent and their parent.

the COVID-19 timepoint when remote learning was occurring, we used four items from the Sleep/Wake Problems subscale in the present study. Specifically, three items were used to create a composite of delayed sleep/wake behaviors (i.e. "stayed up until at least 3 a.m."; "stayed up all night"; "slept in past noon"; $\alpha s=.67$ and .74 before and during COVID-19, respectively). The fourth item was used to assess daytime sleepiness (i.e. "felt tired, dragged out, or sleepy during the day"). In addition, individual items for bedtime, wake time, and numbers of hours slept per night over the prior 2 weeks were used, with separate items used for school days and weekends.

\section{Sleep Disturbance Scale for Children}

The Sleep Disturbance Scale for Children (SDSC) [41] is a 26-item parent-rated scale that evaluates pediatric sleep problems. The scale is well-validated and is one of only two pediatric sleep instruments to meet all criteria for adequate principles of instrument development [42]. Parents completed the seven-item Difficulties Initiating and Maintaining Sleep subscale at both study timepoints; the SDSC instructions ask parents to rate the previous 6 months but this was modified for the COVID-19 assessment point to instruct parents to rate their adolescent's sleep for the previous month. Items are rated on a 5-point scale. The scale queries how many hours of sleep the child gets a night (1=9-11 hours; $5=$ less than 5 hours), how long it takes the child to fall asleep (1=less than $15 \mathrm{~min}$; $5=$ more than $60 \mathrm{~min}$ ), and five items assessing problems with initiating sleep and maintaining sleep (1=never; 5 =always/daily). $T$-scores based on the Bruni et al.[41] sample of 1,157 comparison children and 147 children with sleep problems from Italy were used. T-scores $\geq 70$ are in the clinical range. In the present study, $\alpha$ s $=.75$ and .73 before and during COVID-19, respectively. To examine sleep duration apart from sleep quality, we also examined the sleep duration item separately from a composite mean of the other six items assessing difficulties initiating and maintaining sleep ( $\alpha \mathrm{s}=.77$ at both timepoints).

\section{COVID-19 Adolescent Symptom and Psychological Experience Questionnaire}

The COVID-19 Adolescent Symptom and Psychological Experience Questionnaire (CASPE) [43] is an adolescent-report measure designed to assess experiences and exposures related to COVID-19 and the impact of the COVID-19 pandemic on functioning and was completed by adolescents at the COVID-19 timepoint. In the current study, scales assessing negative affect and difficulties concentrating because of the COVID-19 pandemic were used. COVID-19-related negative affect was assessed using three subscales: worried/afraid (4 items; e.g. anxious, afraid), sad/lonely (5 items; e.g. sad, hopeless, lonely), angry/frustrated (5 items; e.g. irritable, stressed, angry). Items are answered on a 5-point scale in response to how each item was experienced over the prior 7 days due to the COVID-19 pandemic, with mean scores calculated such that higher scores indicate higher levels of negative affect. The difficulties concentrating scale assesses difficulties concentrating and being productive in the prior 7 days due to the COVID-19 pandemic. The scale includes 11 items rated on a 5 -point scale $(1=$ not at all; $5=$ extremely). Example items include, "thinking a lot about COVID-19," "forgetful in daily activities," and "having racing thoughts." Mean scores were calculated to create a difficulty concentrating composite score, with higher scores indicating greater concentration difficulties due to COVID19. In the present study, $\alpha=.90, .78, .86$, and .73 for worried/afraid affect, sad/lonely affect, angry/frustrated affect, and difficulty concentrating due to COVID-19, respectively. 


\section{Coronavirus Health Impact Survey}

The Coronavirus Health Impact Survey (CRISIS) was developed by National Institute of Mental Health (NIMH) investigators and collaborators (www.crisissurvey.org) and was completed by adolescents at the COVID-19 timepoint. In the present study, items from the adolescent version assessing exercise, time outdoors, and social media use were utilized. The exercise item assesses on how many days per week, over the prior 2 weeks, did the adolescent exercise (e.g. increased heart rate, breathing) for at least 30 minutes. The time outdoors item assesses on how many days per week, over the prior 2 weeks, did the adolescent spend time outdoors. The exercise and outdoors items were rated on a five-point scale ( 1 =none; 5 = daily). The social media use item assesses on how much time per day, over the prior 2 weeks, did the adolescent spend using social media (e.g. Instagram, Snapchat, Twitter, TikTok, Facebook). The social media item was rated on a five-point scale ( 1 = no social media; 5 = more than 6 hours).

\section{Analytic Plan}

First, we examined the percentage of adolescents obtaining less than recommended sleep duration (8-10 hours per current National Sleep Foundation Guidelines [39]) on school nights or clinically elevated difficulties initiating and maintaining sleep (per Bruni et al.[41]). These rates were examined across the entire sample and also separately by ADHD status. McNemar's chi-square tests were used to examine whether a significantly higher percentage of adolescents were obtaining sufficient sleep on school nights, or were not experiencing difficulties initiating and maintaining sleep, during the COVID-19 pandemic compared to prior to the COVID-19 pandemic.

Next, linear mixed effect models were conducted using PROC MIXED (SAS version 9.4). This comprehensive modeling approach addressed study objectives 2 and 3 simultaneously by examining a) changes in sleep functioning prior to COVID-19 to during the pandemic across the entire sample, b) comparisons of sleep outcomes between the ADHD and comparison groups across sleep duration, sleep patterns, and delayed sleep/wake behaviors, and c) correlates of sleep duration, difficulties initiating and maintaining sleep, and delayed sleep/ wake behaviors. Specifically, each model includes the effect of time, group, and the interaction between group and time, as well as demographics, medication use, psychiatric comorbidity (including mood disorders, anxiety disorders, and disruptive behavior disorders; see Table 1 note), and the set of correlates for each of the sleep outcomes. These models treated time as a dummy-coded within-person variable such that the main effect for group represents differences between the ADHD and comparison groups across the two timepoints, time represented changes in sleep functioning from prior to COVID-19 to during the pandemic, and Group $\times$ Time interactions represented the effects of ADHD status (relative to non-ADHD comparison adolescents) on changes in sleep functioning from prior to COVID19 to during the pandemic. This modeling approach allowed us to account for the repeated measures obtained from each adolescent across time (i.e. to assess within-person changes in sleep functioning). We used a repeated statement to account for the correlation induced by data collected from the same subject in both timepoints. Models also accounted for clustering effects by site and missing data was handled using full-information maximum likelihood estimates. Models were followed-up with post hoc contrasts comparing the least-squared mean estimates from prior to COVID-19 to during the pandemic, as well as whether adolescents with and without ADHD differed on sleep functioning outcomes prior to and during the pandemic. Cohen's $d$ was calculated as a measure of effect size, with .3, .5 , and.8 representing small, medium, and large effects, respectively (Cohen, 1988).

Finally, the linear fixed effect models allowed us to explore correlates of changes in sleep duration and behaviors during COVID-19. To reduce the number of models being conducted, particularly for this more exploratory aim, we chose a priori to focus on four primary sleep variables pertaining to both parent and adolescent report of sleep duration and quality: parentreported sleep duration, adolescent-reported school night sleep duration, parent-reported difficulties initiating and maintaining sleep, and adolescent-reported delayed sleep/wake behaviors. Health behaviors during COVID-19 (i.e. exercise, spending time outdoors), negative affect during COVID-19 (i.e. worried/afraid, sad/lonely, frustrated/angry), and difficulties concentrating due to COVID-19 were explored as correlates of increased sleep problems. Demographic characteristics (i.e. sex, race, ethnicity, age, family income), medication status (assessed at the preCOVID-19 timepoint), or psychiatric comorbidity variables significantly bivariately correlated with sleep duration or behaviors during COVID-19 were retained as additional correlates for inclusion in the linear mixed models. All variance inflation factor values were below 4.0 and all tolerance values were above .30 (values $>10$ and $<.10$, respectively are typically considered problematic), indicating that multicollinearity was not an issue.

\section{Results}

\section{Changes in clinically recommended sleep duration and clinically elevated sleep difficulties before and during COVID-19}

As indicated in Table 2, the percentage of adolescents obtaining recommended school night sleep duration (8-10 hours) shifted from 57\% before COVID-19 to 64\% during COVID-19, though a McNemar's test indicated that this change was not significantly different $(p=.155)$. As shown in Figure 1, adolescents with ADHD were significantly less likely than adolescents without ADHD to obtain recommended sleep duration on school nights during COVID-19 ( $p=.043)$. Specifically, 59\% of adolescents with ADHD obtained recommended sleep (8-10 hours) during COVID-19, compared to $69 \%$ of adolescents without ADHD (Supplementary Table S3).

We next examined the percentage of adolescents experiencing clinical elevations in parent-reported difficulties initiating and maintaining sleep (i.e. T-scores 270 ). Almost a quarter $(23.7 \%)$ had clinically elevated sleep difficulties before COVID-19, whereas over a third (35.8\%) had clinically elevated sleep difficulties during COVID-19, a significant increase ( $p=.009$; Table 2 ). Whereas only $6 \%$ of the sample had clinically elevated sleep difficulties before COVID-19 and no longer had elevated difficulties during COVID-19, 19\% of the sample did not have elevated difficulties before COVID-19 but did have clinically elevated difficulties during COVID-19. Adolescents with ADHD were more likely to have clinical elevations on this scale both before and during COVID-19 (ps = .041 and .008, respectively; Figure 1). 
Table 2. Changes in clinically recommended sleep duration and clinically elevated sleep difficulties before and during COVID-19

\begin{tabular}{|c|c|c|c|}
\hline Measure & Before COVID-19, \% & During COVID-19, \% & McNemar $\chi^{2}$ Test \\
\hline Clinically elevated difficulties initiating and maintaining sleep & & & $6.76^{* *}$ \\
\hline$T$-score $\geq 70$ & $23.7 \%$ & $35.8 \%$ & \\
\hline T-score $<70$ & $76.3 \%$ & $64.2 \%$ & \\
\hline Clinically recommended sleep duration (school night) & & & 2.03 \\
\hline$<8$ hours & $42.9 \%$ & $33.3 \%$ & \\
\hline $8-10$ hours & $57.1 \%$ & $64.1 \%$ & \\
\hline$\geq 11$ hours & $0.0 \%$ & $2.6 \%$ & \\
\hline Clinically recommended sleep duration (weekend) & & & 0.12 \\
\hline$<8$ hours & $11.7 \%$ & $14.5 \%$ & \\
\hline $8-10$ hours & $73.3 \%$ & $70.1 \%$ & \\
\hline$\geq 11$ hours & $15.0 \%$ & $15.4 \%$ & \\
\hline
\end{tabular}

For McNemar tests for sleep duration, dichotomous variables were used that were recommended sleep (i.e. 8-10 hours) or non-recommended sleep (i.e. $<8$ hours or $\geq 11$ hours)

${ }^{* *} p<.01$.

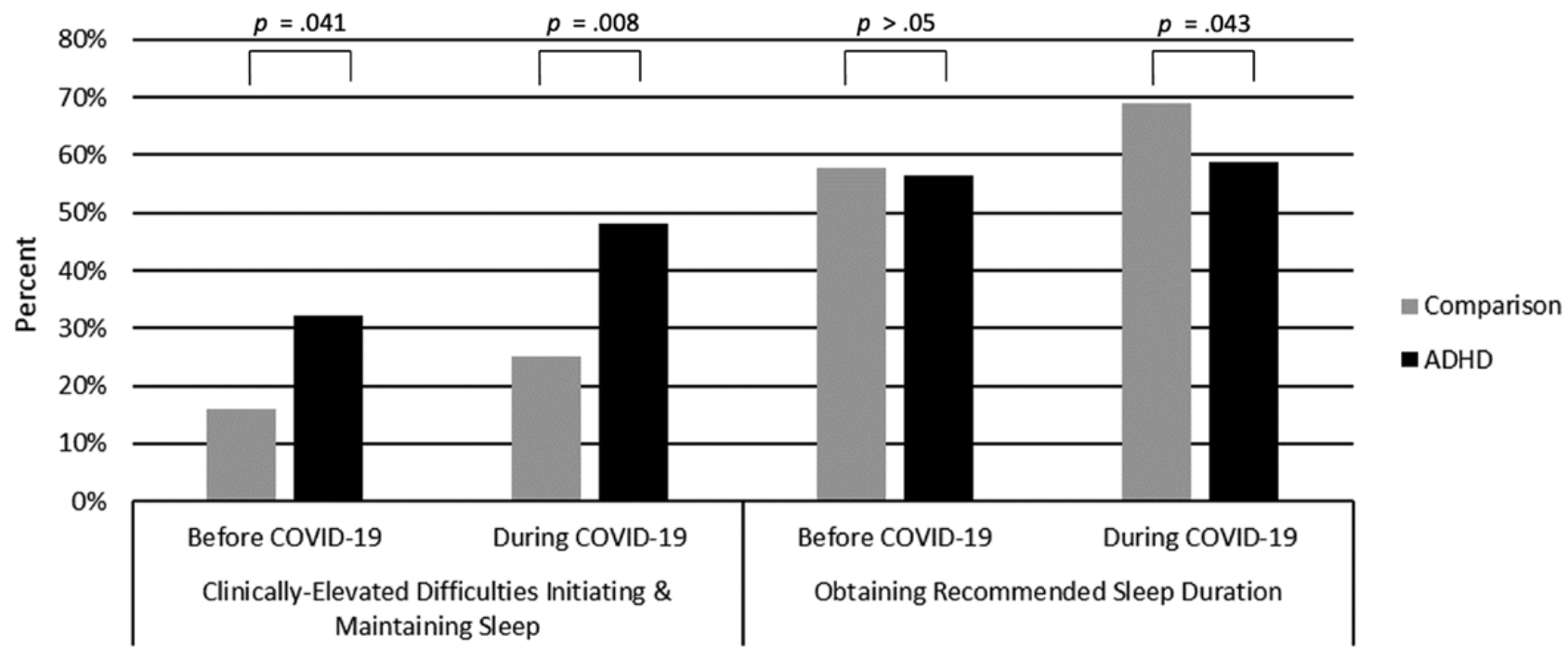

Figure 1. Rates of Clinical Elevations in Difficulties Initiating and Maintaining Sleep and Obtaining Non-Recommended School Night Sleep Duration Before and During COVID-19 in Adolescents with and without ADHD. Clinically elevated difficulties initiating and maintaining sleep (left panel) is the percentage in the clinical range based on the clinical cut-off (T-score $\geq 70$ ) on the Difficulties Initiating and Maintaining Sleep subscale of the Sleep Disturbance Scale for Children. Recommended sleep duration (right panel) is the percentage that were getting recommended sleep duration (i.e. 8-10 hours) on school nights. CC BY 4.0 [Stephen P. Becker].

\section{Changes in sleep/wake schedules before and during COVID-19}

Bedtimes and wake times also significantly shifted from before to during COVID-19, for both school days and weekends. As detailed in Table 3 and displayed in Figure 2, average bedtimes for both school nights and weekends shifted to approximately 1 hour later from pre-COVID-19 to during COVID-19. Average wake times shifted later by over 1.5 hours on school days and 1.2 hours on weekends. Adolescents with and without ADHD did not differ on school night or weekend bedtime or rise time either before or during COVID-19 (Supplementary Table S3).

\section{Changes in sleep duration and behaviors before and during COVID-19 and variables associated with change}

\section{Preliminary analyses}

Descriptive statistics and intercorrelations of demographic characteristics, health behaviors, COVID-19-related negative affect, and difficulties concentrating due to COVID-19 with parent- and adolescent-reported sleep problems were examined to identify a core set of covariates and correlates for the subsequent models (see Supplementary Table S1 for correlations among variables in the full sample and Supplementary Table S2 for intercorrelations separately for the ADHD and comparison groups). Race, family income, medication status, and comorbid diagnosis status were retained as covariates in the primary analyses given significant bivariate associations with sleep problems during COVID-19. Participant sex, ethnicity, age, and social media use were not associated with any of the primary sleep outcomes and were therefore not included in the models. Correlations between time outdoors and exercise and sleep functioning were also negative such that adolescents who reported exercising or spending more time outdoors had fewer parent-reported difficulties initiating and maintaining sleep and adolescent-reported delayed sleep/wake behaviors. Adolescent negative affect (worried, sad/lonely, angry/frustrated) and difficulties concentrating due to COVID-19 were correlated with more difficulties initiating and maintaining sleep and delayed sleep/wake behaviors. 
Table 3. Differences in adolescent sleep/wake patterns, sleep duration and behaviors, and daytime sleepiness before and during COVID-19

\begin{tabular}{|c|c|c|c|c|}
\hline Measure & $\begin{array}{l}\text { Before COVID-19 } \\
\text { Est. M (SE) }\end{array}$ & $\begin{array}{l}\text { During COVID-19 } \\
\text { Est. M (SE) }\end{array}$ & $F$ & $d$ \\
\hline School night bedtime & $10: 44 \mathrm{pm}(.12)$ & $11: 35$ pm (.15) & $49.68^{* * *}$ & .54 \\
\hline Weekend bedtime & $11: 39$ pm (.13) & $12: 37$ am $(.16)$ & $48.42^{* * *}$ & .70 \\
\hline School day rise time & $06: 35$ am (.12) & $08: 13$ am (.12) & $94.84^{* * *}$ & 1.30 \\
\hline Weekend rise time & 09:14 am (.14) & $10: 27$ am (.14) & $70.45^{* * *}$ & .83 \\
\hline PR sleep duration (5-point scale) & $2.74(.08)$ & $2.27(.08)$ & $19.20^{* * *}$ & .55 \\
\hline SR school night sleep duration (hour) & $7.66(.12)$ & $8.05(.11)$ & $8.54^{* *}$ & .32 \\
\hline SR weekend sleep duration (hour) & $9.20(.15)$ & $9.42(.15)$ & 1.64 & .14 \\
\hline PR difficulties initiating and maintaining sleep (T-score) & $61.82(1.06)$ & $65.32(1.06)$ & $16.75^{* * *}$ & .31 \\
\hline SR delayed sleep/wake behaviors & $1.39(.07)$ & $2.05(.07)$ & $58.80^{* * *}$ & .89 \\
\hline SR daytime sleepiness & $2.53(.11)$ & $2.16(.11)$ & $10.31^{* *}$ & .32 \\
\hline
\end{tabular}

Parent-rated sleep duration is rated from 1 (9-11 hours) to 5 (less than 5 hours), such that higher scores represent less sleep duration. Difficulties Initiating and Maintaining Sleep are T-scores from this subscale of the parent-reported Sleep Disturbance Scale for Children (SDSC); all other variables are adolescent-reported on the Sleep Habits Survey (SHS). Estimated means are from linear mixed effects models that accounted for race, family income, medication status, comorbid diagnosis status, time outdoors, exercise, negative affect (worried, sad/lonely, angry/frustrated), and difficulties concentrating due to COVID-19. Effect size $d$ is based on comparisons of linear mixed model estimated means (Est. M) and standard error (SE) estimates which uses repeated measures design that takes the correlation between the two assessment points into account. $\mathrm{PR}=$ parent-report. $\mathrm{SR}=$ adolescent self-report. ${ }^{*} p<.05 .{ }^{* *} p<.01 .{ }^{* * *} p<.001$.

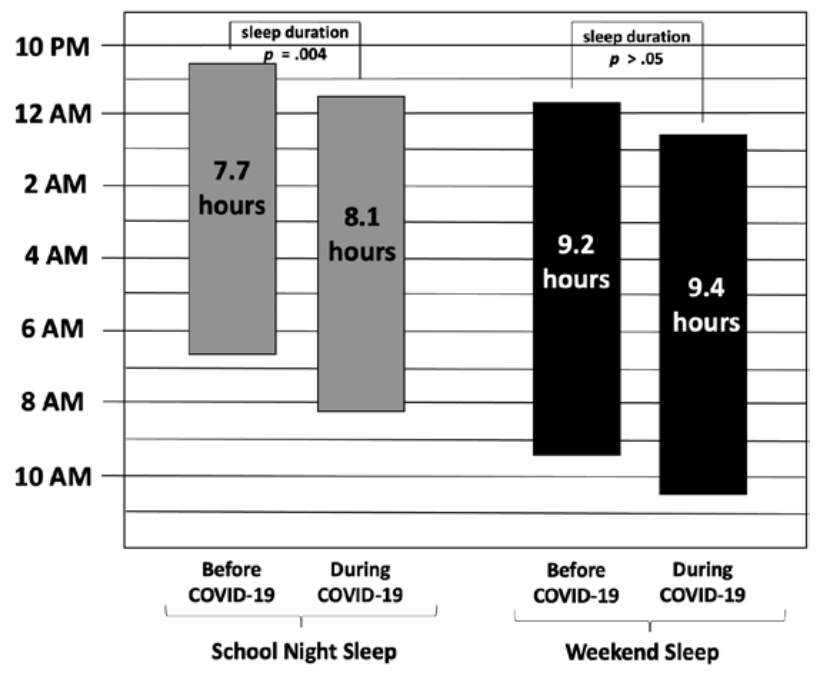

Figure 2. Adolescent Sleep/Wake Patterns Before and During COVID-19. Mean bedtime is represented by the top of each bar, and mean wake time by the bottom of each bar, with average sleep duration printed within each bar. Estimated means are from linear mixed effects models accounted for race, family income, medication status, comorbid diagnosis status, time outdoors, exercise, COVID19-related negative affect (worried, sad/lonely, angry/frustrated), and difficulties concentrating due to COVID-19. CC BY 4.0 [Stephen P. Becker].

\section{Linear mixed effect models}

The effect for each primary sleep variable from before to during COVID-19 across the entire sample are summarized in Table 3. Results of the post hoc contrasts comparing sleep between adolescents with and without ADHD both before and during COVID-19 are summarized in Supplementary Table S1. Results for correlate effects in the linear mixed-effects models for sleep duration and sleep behaviors are presented in Table 4 .

\section{Changes in adolescent sleep duration}

As indicated in Table 3, there was an increase in sleep duration across parent-rated sleep duration $(F=19.20, p<.001)$ and adolescent-rated school night sleep duration $(F=8.54$, $p=.004$ ) from before to during COVID-19. Adolescent ratings of sleep duration on weekend nights did not significantly change from before to during COVID-19 ( $F=1.64, p=.203$ ). There were no significant effects of group or the group by time interaction. Since interactions are underpowered in nonexperimental research [44], we elected to probe the marginal $(F=2.96 ; p=.088)$ group by time interaction for school night sleep duration; pairwise comparisons (Supplementary Table S3) demonstrated that adolescents without ADHD experienced an increase in school night sleep duration $(t=3.36, p=.001)$, whereas adolescents with ADHD did not $(t=0.83, p=.407)$.

Changes in adolescent sleep behaviors and daytime sleepiness As summarized in Table 3, parents reported increased difficulties initiating and maintaining sleep $(F=16.75, p<.001)$ and adolescents reported increased delayed sleep/wake behaviors $(F=58.80, p<.001)$ from before to during COVID-19. As an example of increased delayed sleep/wake behaviors, before COVID-19 only 5.7\% of adolescents reporting staying up until 3 $\mathrm{AM}$ or later at least several times in the past 2 weeks, and this percentage jumped to $28.7 \%$ during COVID-19. Adolescents also reported decreased daytime sleepiness $(F=10.31, p=.002)$ from before to during COVID-19. There were no significant main effects of ADHD group status or the group by time interaction in predicting changes in initiating/maintaining sleep, delayed sleep/wake behaviors, and daytime sleepiness.

\section{Correlates of change in sleep patterns and behaviors}

As summarized in Table 4, after accounting for pre-COVID-19 sleep duration, greater difficulty concentrating due to COVID19 was uniquely associated with parent-reported decreases in sleep duration ( $t=-2.30, p=.023)$. Higher COVID-19-related sad/ lonely affect $(t=-2.01, p=.047)$ and having a comorbid diagnosis $(t=-2.60, p=.011)$ were associated with decreased adolescentreported school night sleep duration.

Spending less time outdoors $(t=-3.17, p=.002)$ and higher worry/afraid affect due to COVID-19 $(t=2.03, p=.045)$ were both uniquely associated with increases in delayed sleep/wake behaviors during COVID-19. Higher sad/lonely affect due to COVID$19(t=1.98, p=.050)$ and having a comorbid diagnosis $(t=4.26, p$ $<.001$ ) were uniquely associated with increased parent-reported difficulties initiating and maintaining sleep. Interestingly, angry/ 
Table 4. Linear mixed models examining COVID-19-related correlates of increased parent- and adolescent-reported sleep duration, delayed sleep/wake behaviors, and difficulties initiating and maintaining sleep during COVID-19

\begin{tabular}{|c|c|c|c|c|c|c|c|c|c|c|c|c|}
\hline & \multicolumn{3}{|c|}{$\begin{array}{l}\text { DV: Parent-reported } \\
\text { sleep duration }\end{array}$} & \multicolumn{3}{|c|}{$\begin{array}{l}\text { DV: Adolescent-reported } \\
\text { school night sleep } \\
\text { duration }\end{array}$} & \multicolumn{3}{|c|}{$\begin{array}{l}\text { DV: Adolescent-reported } \\
\text { delayed sleep/wake } \\
\text { behaviors }\end{array}$} & \multicolumn{3}{|c|}{$\begin{array}{l}\text { DV: Parent-reported } \\
\text { difficulties initiating and } \\
\text { maintaining sleep }\end{array}$} \\
\hline & Est. B & SE & $t$ & Est. B & SE & $t$ & Est. B & SE & $t$ & Est. B & SE & $t$ \\
\hline Time & -.47 & .15 & $-3.05^{* *}$ & .61 & .18 & $3.36^{* * *}$ & .77 & .12 & $6.23^{* * *}$ & .28 & .06 & $4.19^{* * *}$ \\
\hline ADHD status & .16 & .22 & 0.75 & -.36 & .29 & -1.24 & .26 & .18 & 1.43 & .08 & .14 & 0.59 \\
\hline Time $\times$ ADHD & .01 & .22 & 0.02 & -.46 & .26 & $-1.72 \dagger$ & .22 & .17 & 1.31 & .02 & .09 & 0.19 \\
\hline Race & .19 & .20 & 0.94 & .25 & .29 & 0.88 & .06 & .17 & 0.37 & -.03 & .15 & -0.23 \\
\hline Family income & 6.17 & .07 & 0.26 & 4.59 & 3.29 & 1.39 & 5.39 & .20 & 0.27 & -1.63 & 1.69 & -0.97 \\
\hline Medication status & -.07 & .19 & -0.40 & -.16 & .26 & -0.61 & .18 & .16 & 1.15 & -.05 & .13 & -0.35 \\
\hline Comorbidity status & -.20 & .14 & -1.42 & -.52 & .20 & $-2.60^{* *}$ & .09 & .12 & 0.75 & .43 & .10 & $4.26^{* * *}$ \\
\hline Exercise & -.07 & .07 & -1.04 & -.11 & .10 & -1.15 & -.07 & .06 & -1.16 & -.07 & .05 & -1.54 \\
\hline Time spent outdoors & -.10 & .07 & -1.34 & .16 & .10 & 1.58 & -.19 & .06 & $-3.17^{* *}$ & -.04 & .05 & 0.44 \\
\hline Worried/afraid & .15 & .10 & 1.46 & .23 & .15 & 1.56 & .18 & .08 & $2.03^{*}$ & .10 & .08 & 1.31 \\
\hline Sad/lonely & -.06 & .13 & -0.47 & -.37 & .19 & $-2.01^{*}$ & .13 & .11 & 1.1 & .19 & .10 & $1.98^{*}$ \\
\hline Angry/frustrated & .10 & .13 & 0.76 & -.07 & .18 & -0.39 & -.07 & .11 & -0.62 & -.19 & .09 & $-2.06^{*}$ \\
\hline Difficulty concentrating & -.33 & .15 & $-2.30^{*}$ & .22 & .20 & 1.07 & -.04 & .12 & -0.28 & .13 & .10 & 1.26 \\
\hline
\end{tabular}

Parent-rated sleep duration is rated from 1 (9-11 hours) to 5 (less than 5 hours), such that higher scores represent less sleep duration. Parent-reported difficulties initiating and maintaining sleep does not include the sleep duration item. $\mathrm{ADHD}=$ attention-deficit/hyperactivity disorder. For $\mathrm{ADHD}$ status, $0=\mathrm{no} \mathrm{ADHD}, 1=\mathrm{ADHD}$. For race, $0=$ non-White, $1=$ White. $\dagger p<.09 .{ }^{*} p<.05 .{ }^{* *} p<.01 .{ }^{* * *} p<.001$.

frustrated affect was associated with decreased parent-reported sleep difficulties $(t=-2.06, p=.042) .{ }^{1}$

\section{Discussion}

The current study is the first, to our knowledge, to document prospective changes in sleep patterns and behaviors in adolescents before and during the COVID-19 pandemic. Our findings demonstrate that adolescents reported significantly more delayed sleep/wake behaviors and an overall shift to later bedtimes and wake times across both weekdays and weekends during COVID-19 compared to before COVID-19. Adolescents also experienced longer school night sleep duration and less daytime sleepiness during COVID-19, though adolescents with ADHD did not benefit like adolescents without ADHD in terms of increased school night sleep duration during COVID-19 compared to before COVID-19 (it should be noted that while this pairwise comparison was statistically significant, the interaction term itself did not reach statistical significance $[p=.088]$ ). Alongside overall shifts in sleep/wake schedules and longer sleep duration, parents reported that adolescents had more difficulties initiating and maintaining sleep. Finally, this study explored correlates of changes in sleep duration and behaviors, with more worried/afraid affect and less time spent outdoors uniquely associated with more delayed sleep/wake behaviors.

We conducted sensitivity analyses to assess the effects of missing data on the robustness of the association between correlates of parent and adolescent-reported sleep outcomes during the COVID-19 timepoint. Specifically, we fit the linear mixed models including the 16 participants who did not participate in the COVID-19 timepoint. The results were consistent with the models reported in text, with the correlates demonstrating similar effects, and all significant and nonsignificant effects remaining the same, indicating that results of the linear mixed models were not biased due to missing cases at the COVID-19 timepoint.
An important finding from this study is that there may be both positive and negative impacts of the COVID-19 pandemic for adolescent sleep. Particularly during remote learning, adolescents are likely able to adjust their sleep schedules to more closely align with their endogenous circadian phase [4]. Consistent with this possibility, adolescents in the current study had later bedtimes and wake times during COVID-19 compared to before COVID-19. This finding is also consistent with those reported by Leone et al. [5], who in a large sample spanning ages 13-74 found the shift towards later sleep onset and offset to be more pronounced in younger participants. On average, adolescents in the current study woke 1.5 hours later on school days (8:13 AM) during COVID-19 compared to before COVID-19 (6:35 AM), likely reflecting less time needed to get ready in the morning and travel to school. However, it is important to note that while the stated school start time remained consistent before and during COVID-19, we do not have data as to whether adolescents were expected or choose to begin their online learning when the school day started.

Notably, the collective changes in bedtimes and wake times did not curtail sleep duration, as adolescents in the current study obtained more sleep on school nights during than before COVID-19, also consistent with Leone and colleagues [5]. This finding appears to converge with a growing body of research examining school start time for adolescents and recommendations for schools to not start before 08:30 am [45, 46]. Although we did not directly examine whether school start time stayed the same during the pandemic, the later wake time and longer school night sleep duration experienced by adolescents during COVID-19 appear to align with studies implicating school start time (and, by extension, the time it takes to prepare for and travel to school) as a modifiable contributor to insufficient sleep and circadian rhythm disruption in adolescents [45]. However, in the present study, adolescents with ADHD did not experience more school night sleep during COVID-19 and as a result were also less likely than adolescents without ADHD to obtain recommended sleep duration during the pandemic. This is a 
clinically relevant finding given that insufficient sleep duration causes greater inattention, oppositional behaviors, and depressive symptoms in adolescents with ADHD [47, 48]. In considering the findings of the present study, adolescents with ADHD have more challenges with schoolwork, higher family conflict/ stress, greater anxiety and depression, and more technology use than adolescents without ADHD [26, 28, 29, 49]; these are some potential reasons why adolescents with ADHD did not experience the increase in school night sleep duration that was experienced by adolescents without ADHD. In line with this possibility, other data from this sample found that adolescents with ADHD experienced more difficulties with remote learning and had fewer routines during COVID-19, and parents of adolescents with ADHD also experienced more parenting stress than parents of adolescents without ADHD [50]. Additional studies are needed to further evaluate the sleep of adolescents with ADHD during the pandemic, and adolescents with neurodevelopmental disorders such as ADHD may benefit from targeted assessment and intervention to improve sleep during the pandemic and beyond.

Despite changes in sleep schedule and longer school night sleep duration, parents reported that adolescents had increased difficulties initiating and maintaining sleep during COVID-19. In addition, adolescents reported an increase in delayed sleep/ wake behaviors, with over a quarter $(29 \%)$ reporting regularly staying up until $3 \mathrm{AM}$ or later during the pandemic (compared to $6 \%$ before the pandemic). Although adolescents have a later endogenous circadian phase, it is important to recall that the delayed sleep/wake behaviors assessed in this study are part of the Sleep Habits Survey sleep/wake problems scale and reflect more extreme sleep/wake behaviors (i.e. staying up all night, sleeping until noon, staying up until at least $3 \mathrm{AM}$ ). These sleep pattern changes may also be difficult to shift away from as in-person schooling resumes [4].

Considering the study findings collectively, it appears that overall improvements in school night sleep duration may not extend to overall sleep quality. In fact, the percentage of adolescents with clinically elevated difficulties with initiating and maintaining sleep rose $12 \%$ from shortly before to during COVID19. It is possible that there is a 'trade-off' between sleep duration and sleep quality, as experimental research in both adolescents with and without ADHD clearly demonstrates that extending sleep (i.e. longer sleep duration) also contributes to lower sleep efficiency given a decrease in homeostatic sleep drive [47, 51]. In addition, different factors predicted changes in difficulties initiating and maintaining sleep and delayed sleep/wake behaviors during COVID-19. Adolescent-report of experiencing more sadness/loneliness due to COVID-19 was associated with increased parent-report of adolescents' difficulties initiating and maintaining sleep. Studies in adults have also pointed to loneliness and low social support as correlates of sleep problems during COVID-19 [52-54]. This may be especially pronounced in adolescents given the importance of peer relationships and extracurricular activities during this developmental period [55] In addition, experiencing more anger/frustration was uniquely associated with less difficulties initiating and maintaining sleep. Given that the direction of this association flipped from the bivariate correlation analyses, this finding needs replication before interpreting this finding with confidence. Finally, less time spent outdoors and higher reports of COVID-19-related worry/ fear were associated with fewer adolescent-reported sleep problems during COVID-19. Natural sunlight is a strong zeitgeber (entrainment cue) for the circadian system [15], and spending time outside is also associated with greater physical activity and lower stress which may promote sleep [56]. Consistent with this, a study of adults during the COVID-19 pandemic found that being outdoors or in nature was associated with higher positive affect and lower negative affect [57]. In addition, the investigators found that exercising and going for a walk to be daily activities associated with the largest increase in positive affect [57]. It is likely that the variables that we found to be associated with more difficulties and initiating sleep and delayed sleep/ wake behaviors during COVID-19, negative affect and less time outdoors, are themselves intertwined and together are likely to worsen adolescent sleep during the pandemic. Importantly, these factors are also modifiable. Cognitive-behavioral therapy, including behavioral activation, could be useful for improving affect, social connections, and time spent outdoors, which may in turn improve sleep $[58,59]$. In addition, bi-directional processes are likely, and longer sleep duration reduces negative affect in adolescents with and without ADHD [48, 60]. Studies are needed to evaluate whether it is optimal to start intervention using cognitive-behavioral, sleep, or combined interventions, recognizing that the best approach likely depends on the overall clinical picture and case conceptualization.

Strengths of this study include the prospective longitudinal design with assessment points shortly before and during COVID19 , including a sample of adolescents with and without studyassessed ADHD, and examining multiple domains of sleep with both adolescent and parent report. Limitations are also important to note. Only global, subjective sleep measures were used, and the normative information for the SDSC was collected in Italy over 20 years ago. It would be beneficial for future studies to incorporate other methods such as daily sleep diaries and actigraphy. Also, we do not have information regarding specific academic expectations (e.g. homework, exams, class attendance, class schedule), from either parents or teachers, which could relate to sleep behaviors. In addition, our linear mixed models included a variable for psychiatric diagnoses (aside from ADHD), but to reduce the number of independent variables and because of the low rates of any specific diagnosis, we included other psychiatric disorders together in a single variable rather than examining individual disorders, which may be important for understanding the specificity of effects. We also examined health behaviors and emotions concurrently with sleep during COVID-19; it would be informative to examine potential bi-directional associations in studies with multiple timepoints during COVID-19. The present sample was mostly White and from higher income families, and therefore may not generalize to other races, ethnicities, or adolescents in lower-income families. The COVID-19 pandemic is disproportionately impacting Black, Latinx, Native American, and lower-income families [61-63], and disparities in COVID-19related risk may contribute to worsened sleep in these populations. Finally, detailed information regarding medication use (e.g. changes in dosage or timing of medication receipt) was not available at the COVID-19 timepoint; it is important for future research to examine the extent to which medication continuity or discontinuity impacts changes in sleep.

In conclusion, this is the first study to our knowledge to prospectively examine changes in adolescent sleep during the COVID-19 pandemic. The findings shed light on potential positive and negative impacts of the pandemic for adolescent 
sleep. This study focused on initial changes in sleep during the early stages of the COVID-19 pandemic in the United States when stay-at-home orders were in place. It will be important to examine whether changes in sleep persist over time, continue to change, or return to pre-COVID-19 levels as the pandemic continues and ultimately passes. It would also be useful for studies to evaluate whether students attending in-person schooling vs. remote learning have differences in sleep patterns, or whether a hybrid model contributes to more sleep variability which has been linked to poorer functioning [64] and is greater in adolescents with ADHD [65]. Determining whether COVID-19-related changes in sleep predict other negative effects is also important. Given the known benefits of sufficient and high-quality sleep for adolescent health and development $[4,15]$, clinicians should assess and monitor sleep when treating adolescents during the pandemic.

\section{Supplementary material}

Supplementary material is available at SLEEP online.

\section{Funding}

The authors extend their appreciation to the families who participated in this study. This research was conducted at Cincinnati Children's Hospital Medical Center, Cincinnati, Ohio and Virginia Commonwealth University, Richmond, Virginia. This research was supported by a Research Innovation/Pilot award from the Cincinnati Children's Research Foundation (CCRF) and award number R305A160126 from the Institute of Education Sciences (IES), U.S. Department of Education. Stephen Becker and Melissa Dvorsky are supported by grants from the National Institute of Mental Health (NIMH; S.B.: K23MH108603; M.D.: K23MH122839). The content is solely the responsibility of the authors and does not necessarily represent the official views of the CCRF, IES, or NIMH.

Conflict of interest statement. None declared.

\section{Data Availability}

The data underlying this article will be shared on reasonable request to the corresponding author.

\section{References}

1. Pfefferbaum B, et al. Mental Health and the Covid-19 pandemic. N Engl J Med. 2020;383(6):510-512.

2. Gregory AM, et al. Annual Research Review: sleep problems in childhood psychiatric disorders-a review of the latest science. J Child Psychol Psychiatry. 2016;57(3): 296-317.

3. Altena E, et al. Dealing with sleep problems during home confinement due to the COVID-19 outbreak: practical recommendations from a task force of the European CBT-I Academy. J Sleep Res. 2020;29(4):e13052.

4. Becker SP, et al. Editorial perspective: perils and promise for child and adolescent sleep and associated psychopathology during the COVID-19 pandemic. J Child Psychol Psychiatry. 2020;61(7):757-759.

5. Leone MJ, et al. Effects of lockdown on human sleep and chronotype during the COVID-19 pandemic. Curr Biol. 2020;30(16):R930-R931.
6. Cellini $\mathrm{N}$, et al. Changes in sleep pattern, sense of time and digital media use during COVID-19 lockdown in Italy. J Sleep Res. 2020;29(4):e13074.

7. Gupta R, et al. Changes in sleep pattern and sleep quality during COVID-19 lockdown. Indian J Psychiatry. 2020;62(4):370-378.

8. Sinha M, et al. Impact of COVID-19 lockdown on sleep-wake schedule and associated lifestyle related behavior: a national survey. J Public Health Res. 2020;9(3):1826.

9. Li DJ, et al. COVID-19-related factors associated with sleep disturbance and suicidal thoughts among the Taiwanese public: a Facebook survey. Int J Environ Res Public Health. 2020;17(12).

10. Pinto J, et al. Sleep quality in times of Covid-19 pandemic. Sleep Med. 2020;74:81-85.

11. Yu BY, et al. Prevalence of sleep disturbances during COVID-19 outbreak in an urban Chinese population: a cross-sectional study. Sleep Med. 2020;74:18-24.

12. Casagrande $\mathrm{M}$, et al. The enemy who sealed the world: effects quarantine due to the COVID-19 on sleep quality, anxiety, and psychological distress in the Italian population. Sleep Med. 2020;75:12-20.

13. Gualano MR, et al. Effects of Covid-19 lockdown on mental health and sleep disturbances in Italy. Int J Environ Res Public Health. 2020;17(13):4779.

14. Gruber R, et al. The impact of COVID-19 related school shutdown on sleep in adolescents: a natural experiment. Sleep Med. 2020;76:33-35.

15. Crowley SJ, et al. An update on adolescent sleep: new evidence informing the perfect storm model. J Adolesc. 2018;67:55-65.

16. Tarokh L, et al. Sleep in adolescence: physiology, cognition and mental health. Neurosci Biobehav Rev. 2016;70:182-188.

17. Jenni OG, et al. Homeostatic sleep regulation in adolescents. Sleep. 2005;28(11):1446-1454.

18. Zhou SJ, et al. Sleep problems among Chinese adolescents and young adults during the coronavirus-2019 pandemic. Sleep Med. 2020;74:39-47.

19. Beck F, et al.; Coconel Group. Covid-19 health crisis and lockdown associated with high level of sleep complaints and hypnotic uptake at the population level. J Sleep Res. 2021;30(1):e13119.

20. Lin LY, et al. The immediate impact of the 2019 novel coronavirus (COVID-19) outbreak on subjective sleep status. Sleep Med 2021;77:348-354.

21. Voitsidis $\mathrm{P}$, et al. Insomnia during the COVID-19 pandemic in a Greek population. Psychiatry Res. 2020;289:113076.

22. Gao C, et al. Sleep health early in the coronavirus disease 2019 (COVID-19) outbreak in the United States: integrating longitudinal, cross-sectional, and retrospective recall data. Sleep Med. 2020;73:1-10.

23. Breaux R, et al. ADHD in COVID-19: Risk, resilience, and the rapid transition to telehealth. The ADHD Report. 2021;29:1-10.

24. Becker SP, et al. Sleep and daytime sleepiness in adolescents with and without ADHD: differences across ratings, daily diary, and actigraphy. J Child Psychol Psychiatry. 2019;60(9):1021-1031.

25. Lunsford-Avery JR, et al. Sleep disturbances in adolescents with ADHD: A systematic review and framework for future research. Clin Psychol Rev. 2016;50:159-174.

26. Bourchtein E, et al. Technology use and sleep in adolescents with and without attention-deficit/hyperactivity disorder. Journal of pediatric psychology. 2019;44(5):517-526.

27. Deault LC. A systematic review of parenting in relation to the development of comorbidities and functional impairments 
in children with attention-deficit/hyperactivity disorder (ADHD). Child Psychiatry Hum Dev. 2010;41(2):168-192.

28. Becker SP, et al. Psychiatric co-occurrence (comorbidity) in adolescents with ADHD. In: Becker SP, ed. ADHD in Adolescents: Development, Assessment, and Treatment. New York, NY: Guilford; 2020:170-203.

29. Jarrett MA, et al. A conceptual review of the comorbidity of attention-deficit/hyperactivity disorder and anxiety: implications for future research and practice. Clin Psychol Rev. 2008;28(7):1266-1280.

30. Zhang J, et al. Acute stress, behavioural symptoms and mood states among school-age children with attentiondeficit/hyperactive disorder during the COVID-19 outbreak. Asian J Psychiatr. 2020;51:102077.

31. Sciberras E, et al. Physical health, media use and mental health in children and adolescents with ADHD during the COVID-19 pandemic in Australia. J Atten Disor. 2020. doi:10.1177/1087054720978549

32. Moore SA, et al. Impact of the COVID-19 virus outbreak on movement and play behaviours of Canadian children and youth: a national survey. Int $J$ Behav Nutr Phys Act. 2020;17(1):85.

33. Klaiber $P$, et al. The ups and downs of daily life during COVID-19: Age differences in affect, stress, and positive events. J Gerontol B Psychol Sci Soc Sci. 2021;76:e30-e37.

34. Brose A, et al. Change in Mental Health Symptoms During the COVID-19 Pandemic: The Role of Appraisals and Daily Life Experiences. J Pers. 2020.

35. Bäuerle A, et al. Mental Health Burden of the COVID19 Outbreak in Germany: Predictors of Mental Health Impairment. J Prim Care Community Health. 2020;11:2150132720953682.

36. Son C, et al. Effects of COVID-19 on College Students' Mental Health in the United States: Interview Survey Study. J Med Internet Res. 2020;22(9):e21279.

37. Orgilés $\mathrm{M}$, et al. Immediate Psychological Effects of the COVID-19 Quarantine in Youth From Italy and Spain. Front Psychol. 2020;11:579038.

38. Xiao $\mathrm{H}$, et al. Social capital and sleep quality in individuals who self-isolated for 14 days during the Coronavirus Disease 2019 (COVID-19) outbreak in January 2020 in China. Med Sci Monit. 2020;26:e923921.

39. Hirshkowitz M, et al. National Sleep Foundation's sleep time duration recommendations: methodology and results summary. Sleep Health. 2015;1(1):40-43.

40. Wolfson AR, et al. Sleep schedules and daytime functioning in adolescents. Child Dev. 1998;69(4):875-887.

41. Bruni O, et al. The Sleep Disturbance Scale for Children (SDSC). Construction and validation of an instrument to evaluate sleep disturbances in childhood and adolescence. J Sleep Res. 1996;5(4):251-261.

42. Spruyt K, et al. Pediatric sleep questionnaires as diagnostic or epidemiological tools: a review of currently available instruments. Sleep Med Rev. 2011;15(1):19-32.

43. Ladouceur CD. COVID-19 Adolescent Symptom and Psychological Experience Questionnaire (CASPE). Pittsburgh, PA: Author; 2020.

44. McClelland $\mathrm{GH}$, et al. Statistical difficulties of detecting interactions and moderator effects. Psychol Bull. 1993;114(2):376-390.

45. American Academy of Pediatrics. School start times for adolescents. Pediatrics. 2014;134(3):642-649.

46. Watson NF, et al.; American Academy of Sleep Medicine Board of Directors. Delaying Middle school and high school start times promotes student health and performance: an American Academy of Sleep Medicine Position Statement. J Clin Sleep Med. 2017;13(4):623-625.

47. Becker SP, et al. Shortened sleep duration causes sleepiness, inattention, and oppositionality in adolescents with attention-deficit/hyperactivity disorder: findings from a crossover sleep restriction/extension study. J Am Acad Child Adolesc Psychiatry. 2019;58(4):433-442.

48. Becker SP, et al. Impact of sleep restriction on affective functioning in adolescents with attention-deficit/hyperactivity disorder. J Child Psychol Psychiatry. 2020;61(10):1160-1168.

49. Becker SP, ed. ADHD in Adolescents: Development, Assessment, and Treatment. New York, NY: Guilford; 2020.

50. Becker SP, et al. Remote learning during COVID-19: examining school practices, service continuation, and difficulties for adolescents with and without attention-deficit/hyperactivity disorder. J Adolesc Health. 2020;67(6):769-777.

51. Beebe DW, et al. Feasibility and behavioral effects of an at-home multi-night sleep restriction protocol for adolescents. J Child Psychol Psychiatry. 2008;49(9):915-923.

52. Grossman ES, et al. COVID-19 related loneliness and sleep problems in older adults: Worries and resilience as potential moderators. Pers Individ Dif. 2021;168:110371.

53. Grey I, et al. The role of perceived social support on depression and sleep during the COVID-19 pandemic. Psychiatry Res. 2020;293:113452.

54. Kokou-Kpolou CK, et al. Insomnia during COVID-19 pandemic and lockdown: Prevalence, severity, and associated risk factors in French population. Psychiatry Res. 2020;290:113128.

55. Brown BB, Larson JA. Peer relationships in adolescence. In: Lerner RM, Steinberg L, eds. Handbook of Adolescent Psychology: Contextual Influences on Adolescent Development. Hoboken, New Jersey: John Wiley \& Sons; 2009:74-103.

56. Sterdt E, et al. Correlates of physical activity of children and adolescents: A systematic review of reviews. Health Education Journal. 2014;73(1):72-89.

57. Lades LK, et al. Daily emotional well-being during the COVID-19 pandemic. BrJ Health Psychol. 2020;25(4):902-911.

58. Conroy DA, et al. Modified cognitive behavioral therapy for insomnia in depressed adolescents: a Pilot Study. Behav Sleep Med. 2019;17(2):99-111.

59. Harvey AG, et al. Modifying the impact of eveningness chronotype ("Night-Owls") in youth: a randomized controlled trial. J Am Acad Child Adolesc Psychiatry. 2018;57(10):742-754.

60. Baum KT, et al. Sleep restriction worsens mood and emotion regulation in adolescents. J Child Psychol Psychiatry. 2014;55(2):180-190.

61. Garg S. Hospitalization rates and characteristics of patients hospitalized with laboratory-confirmed coronavirus disease 2019-COVID-NET, 14 States, March 1-30, 2020. Morb Mortal Wkly Rep. 2020;69:458-464.

62. Tai DBG, et al. The disproportionate impact of COVID-19 on racial and ethnic minorities in the United States. Clin Infect Dis. 2021;72(4):703-706.

63. Raifman MA, et al. Disparities in the population at risk of severe illness from COVID-19 by race/ethnicity and income. Am J Prev Med. 2020;59(1):137-139.

64. Becker SP, et al. Intraindividual variability of sleep/wake patterns in relation to child and adolescent functioning: a systematic review. Sleep Med Rev. 2017;34:94-121.

65. Langberg $\mathrm{JM}$, et al. Intraindividual variability of sleep/ wake patterns in adolescents with and without attentiondeficit/hyperactivity disorder. J Child Psychol Psychiatry. 2019;60(11):1219-1229. 